\title{
Optimising the LHCb VELO detector
}

\author{
M. McCubbin \\ Liverpool University
}

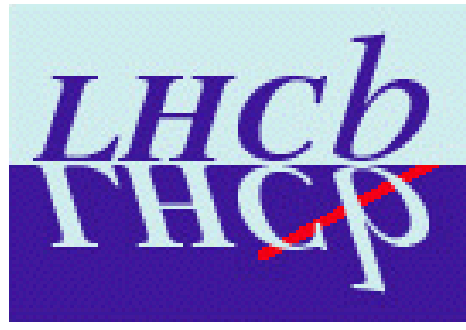

VERTEX2000, 10th - 15th September, 2000

\section{Outline:}

- LHCb overview

- VELO tasks

- VELO layout

- Required VELO performance

- Updates to VELO design

- Simulation of new VELO designs

- Outlook 


\section{LHCb overview}

Aim: study CP violation in $B$ meson decays

LHC: $p p$ collisions at c.o.m. energy $14 \mathrm{TeV}$

$L=2.0 \times 10^{32} \mathrm{~cm}^{-2} \mathrm{~s}^{-1}$

bunch-crossing (interaction) rate 40 (15) $\mathrm{MHz}$

$\sigma_{\text {inel }}=100 \mathrm{mb}, \sigma_{\mathrm{b} \overline{\mathrm{b}}}=500 \mu \mathrm{b}$

$\rightarrow 10^{12} \mathrm{~b} \overline{\mathrm{b}}$ produced per LHC year $\left(10^{7} \mathrm{~s}\right)$

$\rightarrow$ high statistics samples for precision

measurements of CP asymmetries, $B$

oscillations and rare $B$ decay studies

Experimental needs:

a) efficient trigger for leptons, hadrons and displaced vertices ( $B$ meson selection)

b) particle ID ( $\pi / \mathrm{K}$ separation, flavour tagging)

c) good mass resolution (background rejection)

d) good decay time resolution $\left(\Delta \mathrm{m}_{s}, \Delta \Gamma_{s}\right)$ 


\section{LHCb overview (cont'd)}

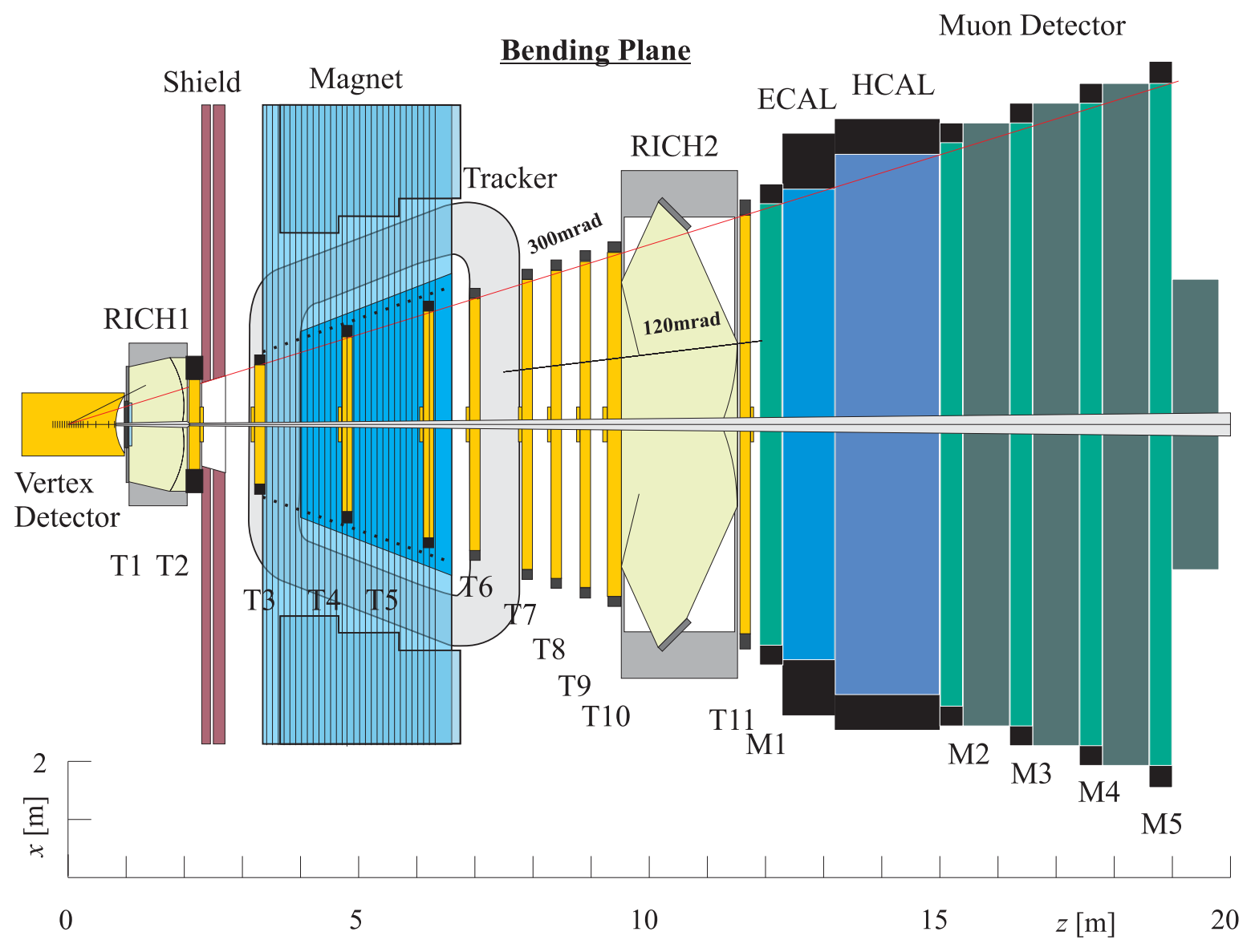

Single-arm spectrometer covering $\theta=$

$15 \mathrm{mrad}$ to $300 \mathrm{mrad}(\eta=1.88$ to 4.89$)$

Main features:

VErtex LOcator (VELO) - vertex detector

usual complement of tracking chambers, $\mathrm{RICH}$,

ECAL, HCAL, muon chambers and magnet 


\section{VELO tasks}

VELO has 2 main tasks:

a) precise track measurement near interaction region $\longrightarrow$ good resolution on track impact

parameters (IP), primary (PV) and secondary (SV) vertices and $B$ decay length

b) use in LHCb trigger to enrich $B$ sample

L0: input rate $40 \mathrm{MHz}$, output rate $1 \mathrm{MHz}$ based on high $p_{t}$ leptons, hadrons and photons + "pile-up" veto

L1: input rate $1 \mathrm{MHz}$, output rate $40 \mathrm{kHz}$ based on vertex (VELO) and track triggers

L2 and L3 reduce rate to $200 \mathrm{~Hz}$ (final sample mostly $b \bar{b}, c \bar{c}$ events) 


\section{VELO layout}

\section{At time of Technical Proposal (1998)...}
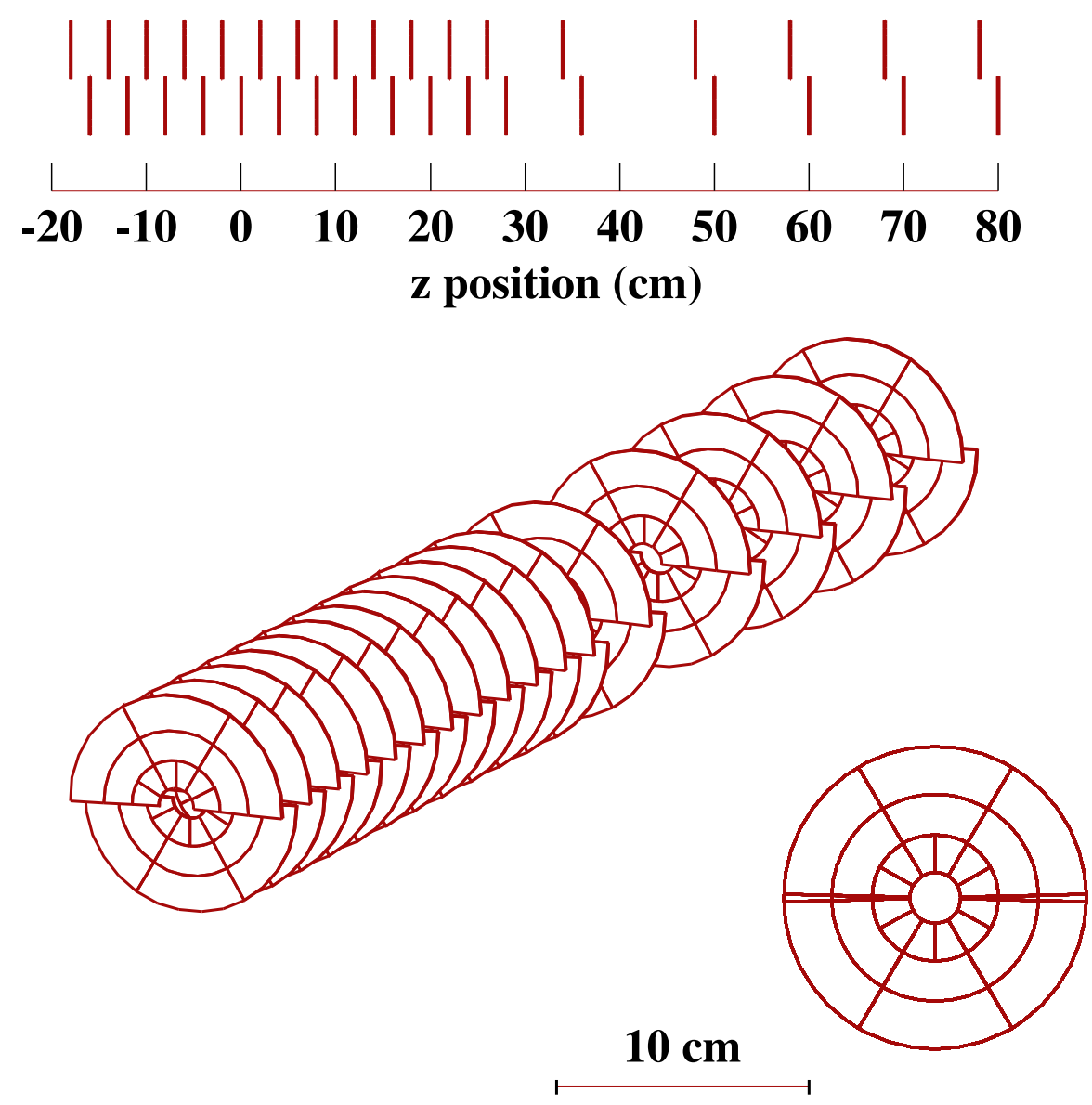

17 disks (stations) of $r$ and $\phi$ detectors

upper-lower halves retract during LHC injection

upper-lower overlap for alignment 


\section{VELO layout (cont'd)}

Strip layout:

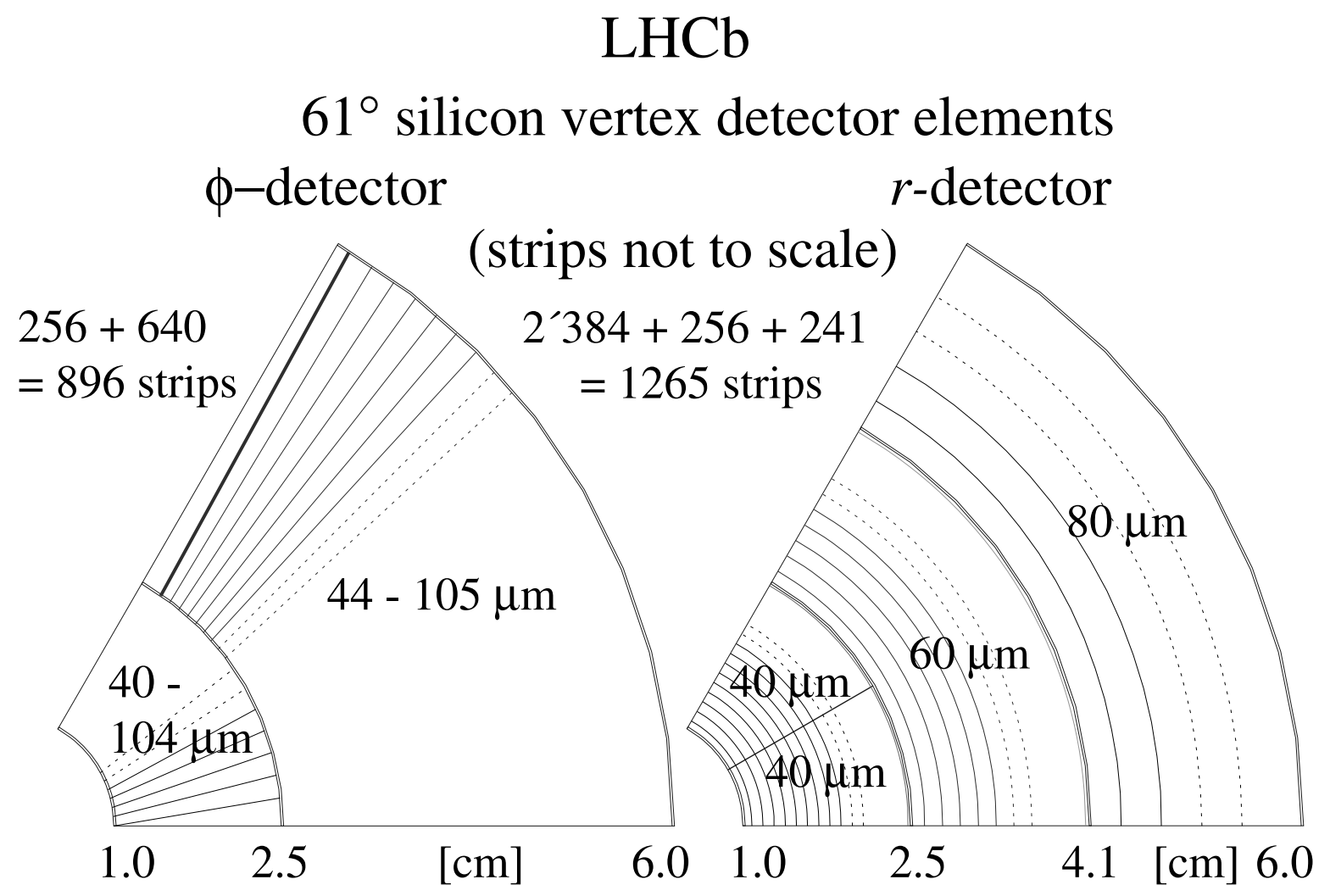

Si thickness $=150 \mu \mathrm{m}$

module overlap for alignment

channel occupancy less than $0.5 \%$ 


\section{VELO layout (cont'd)}

RF shielding:

beam in primary LHC vacuum, detector and electronics protected from beam RF pick-up in secondary vacuum

Al (100 $\mu$ m thick) caps round Si

Al thick - withstand any differential pressure, provide enough shielding

Al thin - not degrade resolution

Vacuum tank:

VELO mounted on support structures in cylindrical vacuum tank

alignment, retraction of VELO, mechanical stress from heat load, maintain vacuum with signal feed-throughs

low mass in acceptance region 


\section{VELO layout (cont'd)}

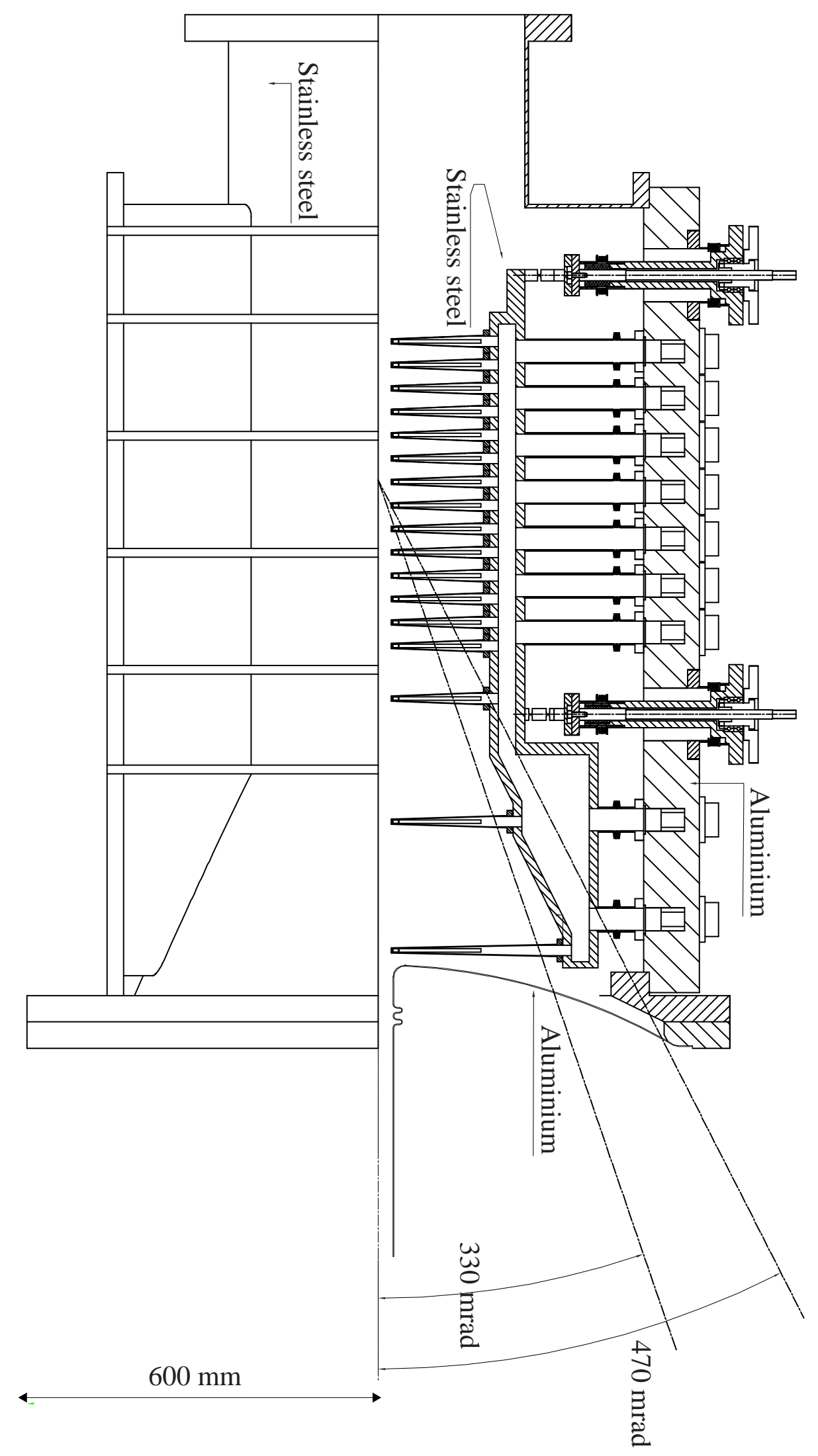




\section{Required VELO performance}

Simulated resolutions:

primary vertex $-\sigma=40 \mu \mathrm{m}$

secondary vertex $-\sigma=180 \mu \mathrm{m}(B \rightarrow \pi \pi)$
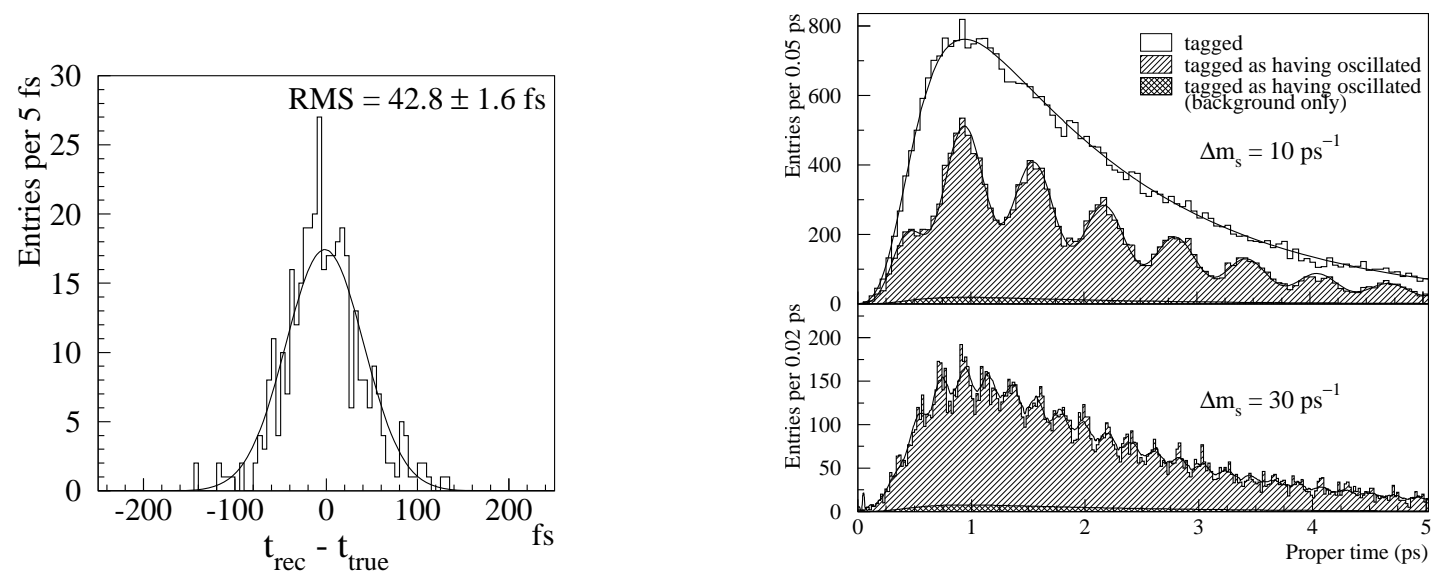

proper-time resolution for $B_{s} \rightarrow D_{s}^{-} \pi^{+}$

events $=43 \mathrm{fs} \longrightarrow 5 \sigma$ measurement of $\Delta \mathrm{m}_{s}$ up to $48 \mathrm{ps}^{-1}$ (in one year) 


\section{Required VELO performance (cont'd)}

L1 vertex trigger:

a) 2D track search in $r-z$ projection

b) estimate of PV using 2D tracks

c) for $2 \mathrm{D}$ tracks not from $\mathrm{PV}$ add $\phi$ information

$\rightarrow$ 3D tracks

d) search for SV using pairs of 3D tracks

output - probability based on number of SV and distance from $\mathrm{PV}$

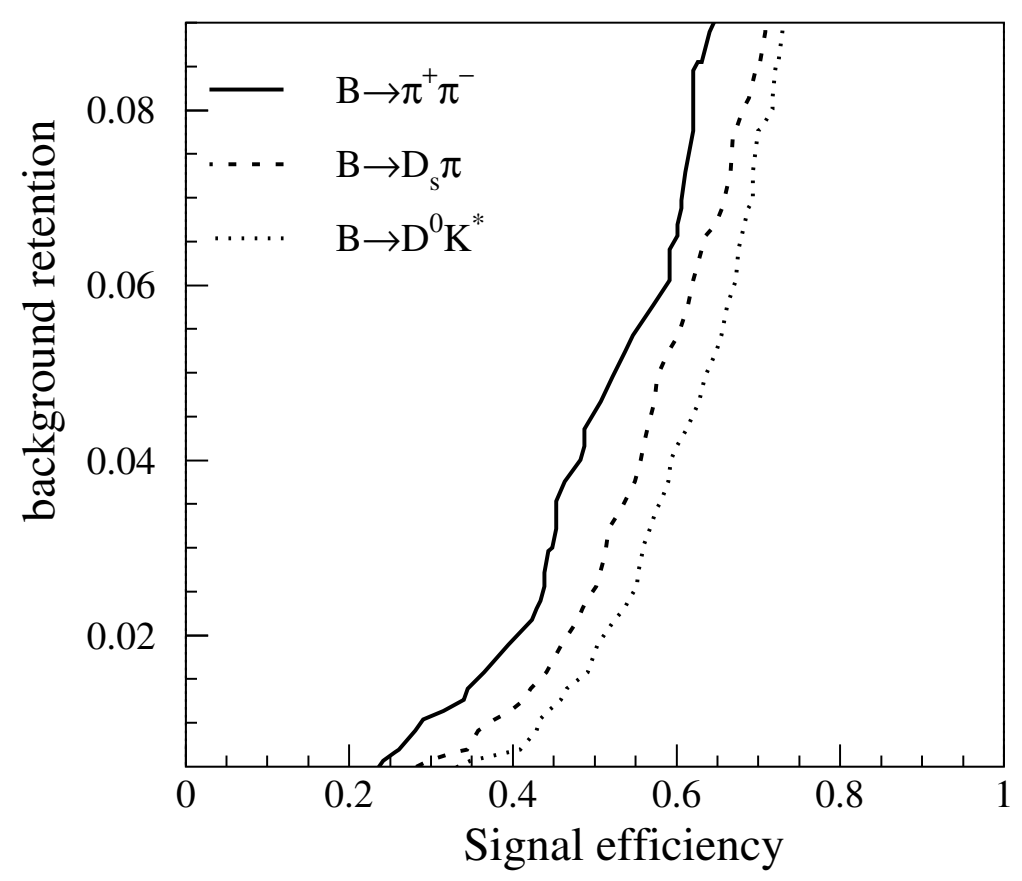




\section{Updates to VELO design}

Si thickness:

thin - decrease depletion voltage, lower bulk leakage current (less cooling required), reduced signal and $\mathrm{S} / \mathrm{N}$ needing better electronics

thick - increased multiple scattering, larger operating voltage $\rightarrow$ breakdown, more heating availability (thickness) depends on technology and manufacturer - p-in-n generally thinner and cheaper than n-in-n

choice: $220 \mu \mathrm{m}$ with $300 \mu \mathrm{m}$ as backup

(TP: $150 \mu \mathrm{m}$ ) 


\section{Updates to VELO design (cont'd)}

Strip pitch:

hit resolution $\approx \mathrm{SP} / 12$ with low noise analog

electronics and charge-sharing

technology choice affects SP -

n-strip with p-stops: min. $40 \mu \mathrm{m}$

p-strip: min. $20-25 \mu \mathrm{m}$

resolutions from simulation studies -
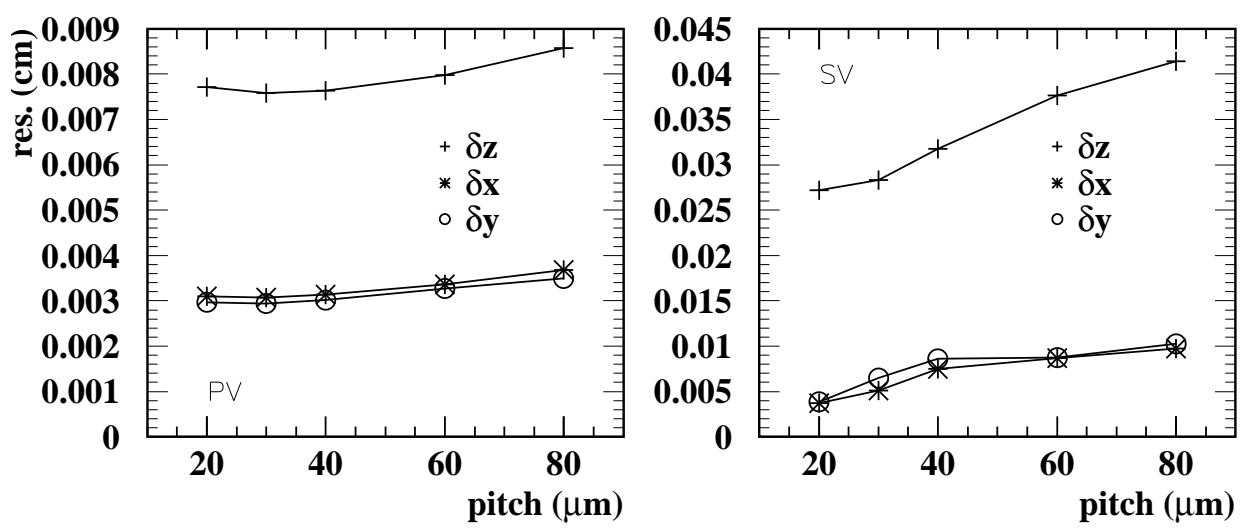

$\rightarrow$ strip pitch important for SV resolution

choice: 20/30/40 $\mu \mathrm{m}$ for $r$ detectors (backup

as TP: $40 / 60 / 80 \mu \mathrm{m})$ 


\section{Updates to VELO design (cont'd)}

Si inner/outer radius:

reducing inner radius improves IP resolution

$\rightarrow$ reduce outer radius to maintain number of electronics channels

6"/4" facilities and flexibility of options -

$180^{\circ}$ sensors on 4 " wafers: max. radius $4.5 \mathrm{~cm}$ not all manufacturers moved to 6"

choice: IR $0.8 \mathrm{~cm}$, OR $4.5 \mathrm{~cm}$ (TP: $1.0 \mathrm{~cm}$ and $6.0 \mathrm{~cm})$ 


\section{Updates to VELO design (cont'd)}

Number of Si stations:

tracks in LHCb - at least VELO 3 hits

increase from 17 - further improve tracking

capabilities $\longrightarrow$ cost, complexity, space

fast optimisation -

PYTHIA+beam-spot+MS+inefficiencies

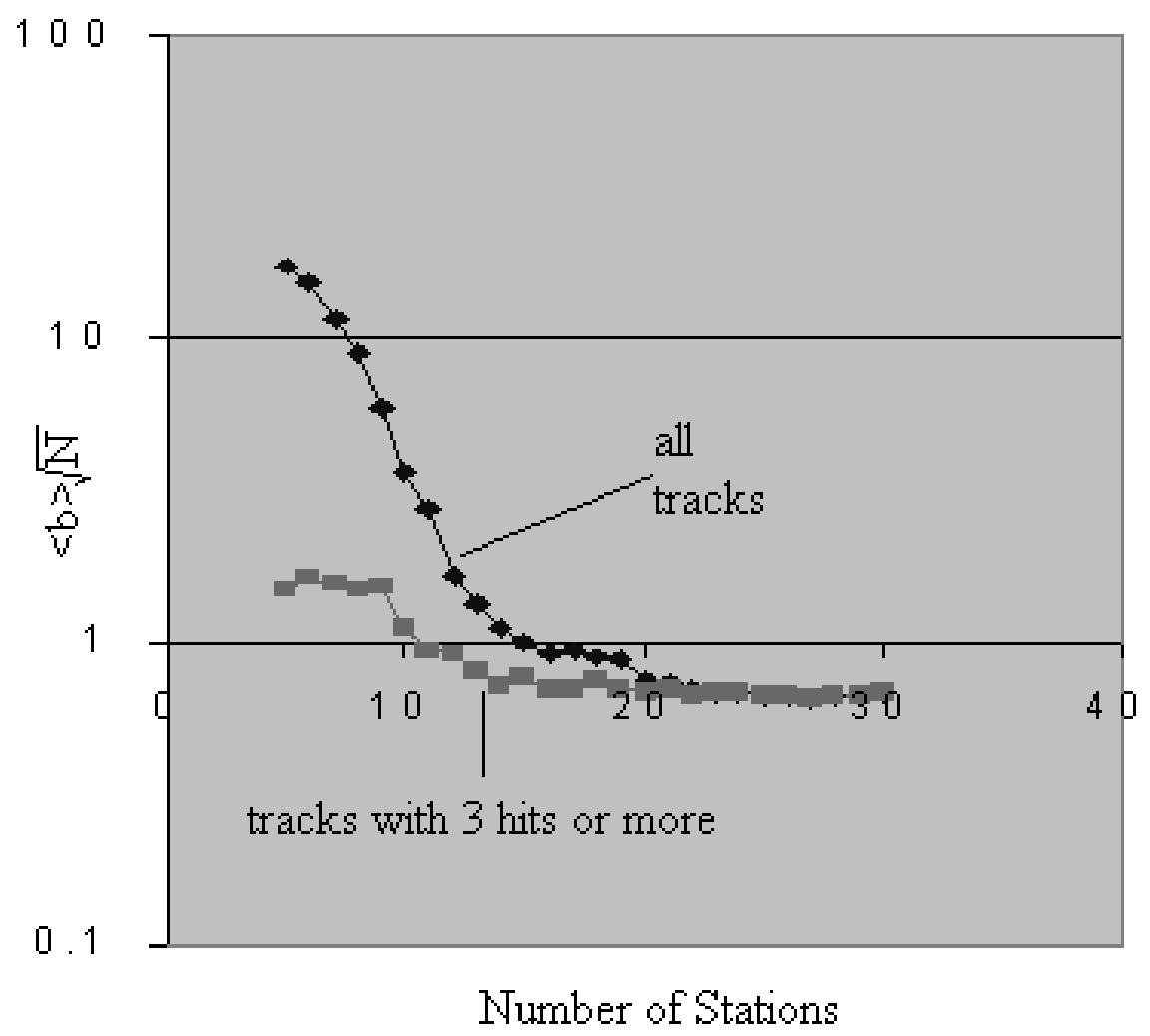

choice: 25 stations (TP: 17) 


\section{Updates to VELO design (cont'd)}

RF design:

TP shielding thickness not sufficient

wake field suppressors needed -4 strips

along VELO length $\rightarrow$

problems - thickness, cooling, mechanics
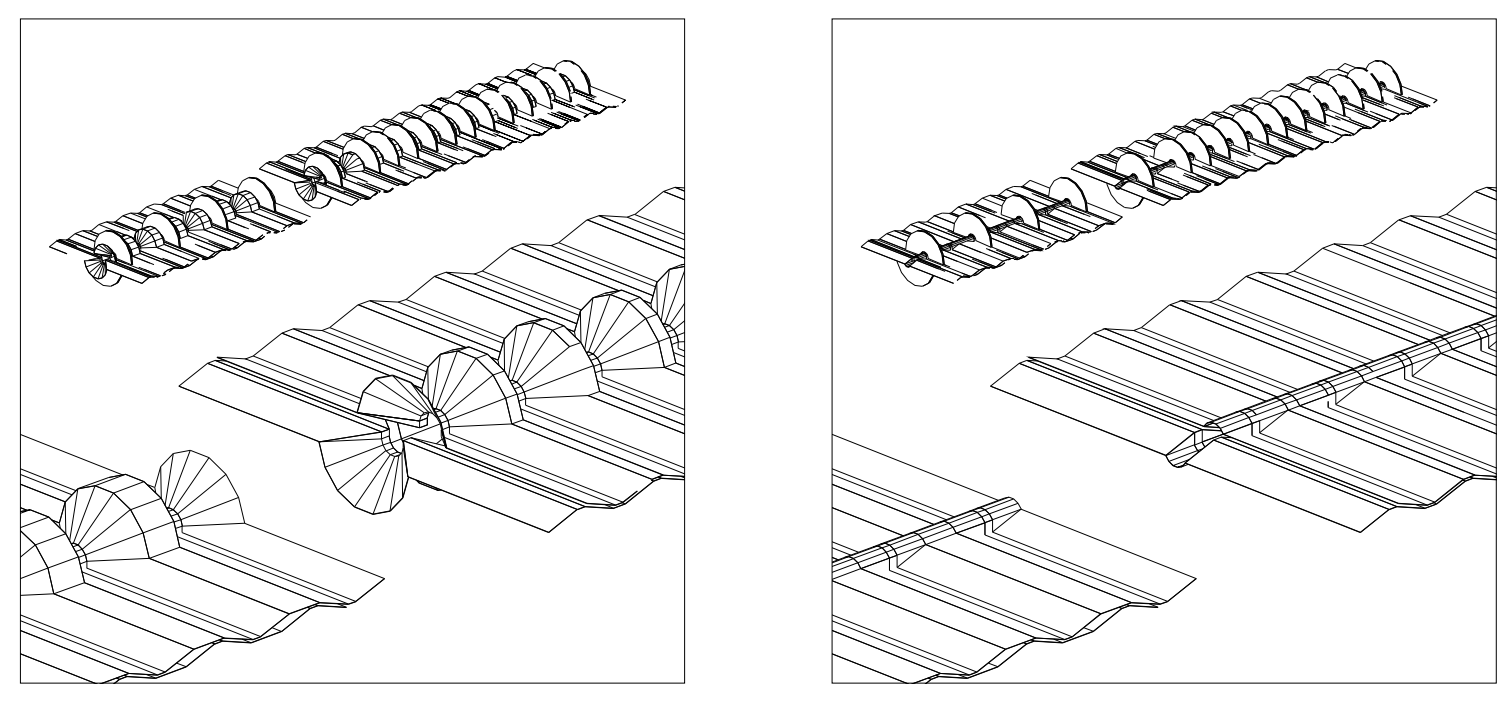

Al box acts as wake field suppressor

corrugation needed for upper-lower overlap

minimise multiple scattering

choice: "toblerone" design, Al $250 \mu \mathrm{m}$ (TP:

$100 \mu \mathrm{m})$ 


\section{Updates to VELO design (cont'd)}

Si radiation tolerance:

extreme environment — damage degrades

performance

technology — n-in-n more "rad. hard"

results from irradiated detectors + work with

oxygenated detectors, thinner detectors

$\rightarrow$ better understanding of effects allowing $\mathrm{Si}$

closer to beam

Overall material budget:

minimise effect of changes on rest of $\mathrm{LHCb}$ 


\section{Simulation of new VELO designs}

Simulated new VELO design parameters with standard LHCb simulation (SICB: PYTHIA + GEANT)

Use MAP facility at Liverpool (http://www.ph.liv.ac.uk/map/)

Quantities investigated:

RMS distance between the true and reconstructed PV

RMS distance between the true and reconstructed SV double-Gaussian fits to (true - rec.) decay lengths

$B$ decay selection efficiencies and backgrounds charged and neutral particle multiplicities number of hits per event in other LHCb detectors number of VELO hits per track particle flux versus radius

L0 selection efficiency number of high IP tracks - for L1

L1 efficiency versus min. bias retention 


\section{Simulation of new VELO designs (cont'd)}

Multiparameter problem — investigate

performance for many combinations of VELO

design parameters

$\rightarrow$ large event samples used for this study:

\begin{tabular}{|ccc|}
\hline study & event type & n. events \\
\hline \hline resolution & $B \rightarrow \pi \pi$ & $378 \mathrm{k}$ \\
& $B \rightarrow J / \psi(\mu \mu) K_{s}^{0}$ & $440 \mathrm{k}$ \\
\hline event selection & $B \rightarrow \pi \pi$ & $771 \mathrm{k}$ \\
& $B \rightarrow J / \psi(\mu \mu) K_{s}^{0}$ & $730 \mathrm{k}$ \\
\hline trigger, multiplicity & $B \rightarrow \pi \pi$ & $364 \mathrm{k}$ \\
and other & minimum bias & $987 \mathrm{k}$ \\
\hline
\end{tabular}

Over 10 million fully simulated LHCb events in total for this and other studies 


\section{Simulation of new VELO designs (cont'd)}

\begin{tabular}{|c|c|c|c|c|c|c|}
\hline \multirow[b]{2}{*}{$\begin{array}{l}\text { VELO } \\
\text { design }\end{array}$} & \multirow[b]{2}{*}{$\begin{array}{l}\text { number } \\
\text { stations }\end{array}$} & \multicolumn{2}{|c|}{ Si parameters } & \multirow{2}{*}{$\begin{array}{c}\text { strip-pitch } \\
\text { SPir/SPmr/SPor } \\
(\mu \mathrm{m})\end{array}$} & \multicolumn{2}{|c|}{ RF shield } \\
\hline & & $\begin{array}{c}\mathrm{d} \\
(\mu \mathrm{m})\end{array}$ & $\begin{array}{l}\text { IR/OR } \\
(\mathrm{cm})\end{array}$ & & design & $\begin{array}{l}\text { ALth } \\
(\mu \mathrm{m})\end{array}$ \\
\hline TP & 17 & 150 & $1.0 / 6.0$ & $40 / 60 / 80$ & TP & $\begin{array}{l}100 \\
250\end{array}$ \\
\hline I & 25 & 220 & $1.0 / 4.5$ & $40 / 60 / 80$ & TP & $\begin{array}{l}100 \\
250\end{array}$ \\
\hline la & 25 & 220 & $0.8 / 4.5$ & $40 / 60 / 80$ & TP & $\begin{array}{l}100 \\
250\end{array}$ \\
\hline II & 17 & 220 & $1.0 / 6.0$ & $40 / 60 / 80$ & tb & $\begin{array}{l}100 \\
250\end{array}$ \\
\hline III & 17 & 220 & $1.0 / 6.0$ & $40 / 60 / 80$ & bp & $\begin{array}{l}100 \\
250\end{array}$ \\
\hline IV & 25 & 220 & $0.8 / 4.5$ & $20 / 30 / 40$ & TP & $\begin{array}{l}100 \\
250\end{array}$ \\
\hline Va & 25 & 220 & $0.8 / 4.5$ & $40 / 60 / 80$ & tb & 250 \\
\hline $\mathrm{Vb}$ & 25 & 220 & $0.8 / 4.5$ & $20 / 30 / 40$ & tb & 250 \\
\hline baseline & 25 & 220 & $0.8 / 4.5$ & $20 / 30 / 40$ & tb & 250 \\
\hline backup & 25 & 300 & $0.8 / 4.5$ & $40 / 60 / 80$ & tb & 250 \\
\hline
\end{tabular}

tb - "toblerone" RF, bp — "beampipe" RF + TP with Si $500 \mu \mathrm{m}$ thick 


\section{Simulation of new VELO designs (cont'd)}

Particle fluxes for min. bias:
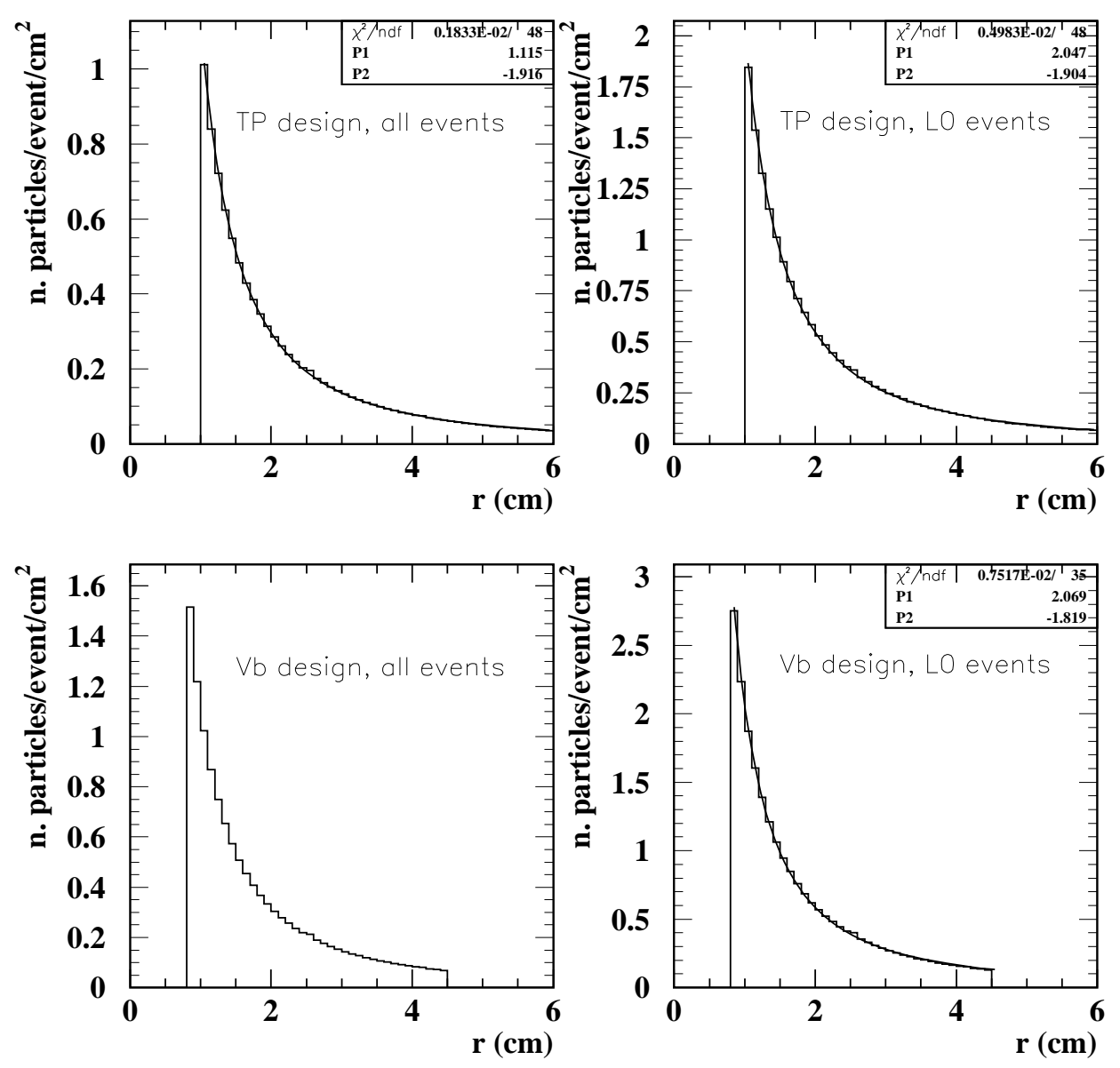

$\sigma_{\text {inel }}, L$ and $1 \mathrm{LHC}$ year as before:

$1.6 \times 10^{14} \mathrm{~cm}^{-2}$ charged particles/year

Mostly pions, fold with $p$ and NIEL constants:
$0.6-1.1 \times 10^{14} \mathrm{~cm}^{-2} 1 \mathrm{MeV}$ neutrons/year
(at $r=1 \mathrm{~cm}$, depending on $z$ ) 


\section{Simulation of new VELO designs (cont'd)}

Event selection efficiencies and backgrounds for $B \rightarrow \pi \pi$ and $B \rightarrow J / \psi(\mu \mu) K_{s}^{0}$ :

event selection efficiencies and backgrounds
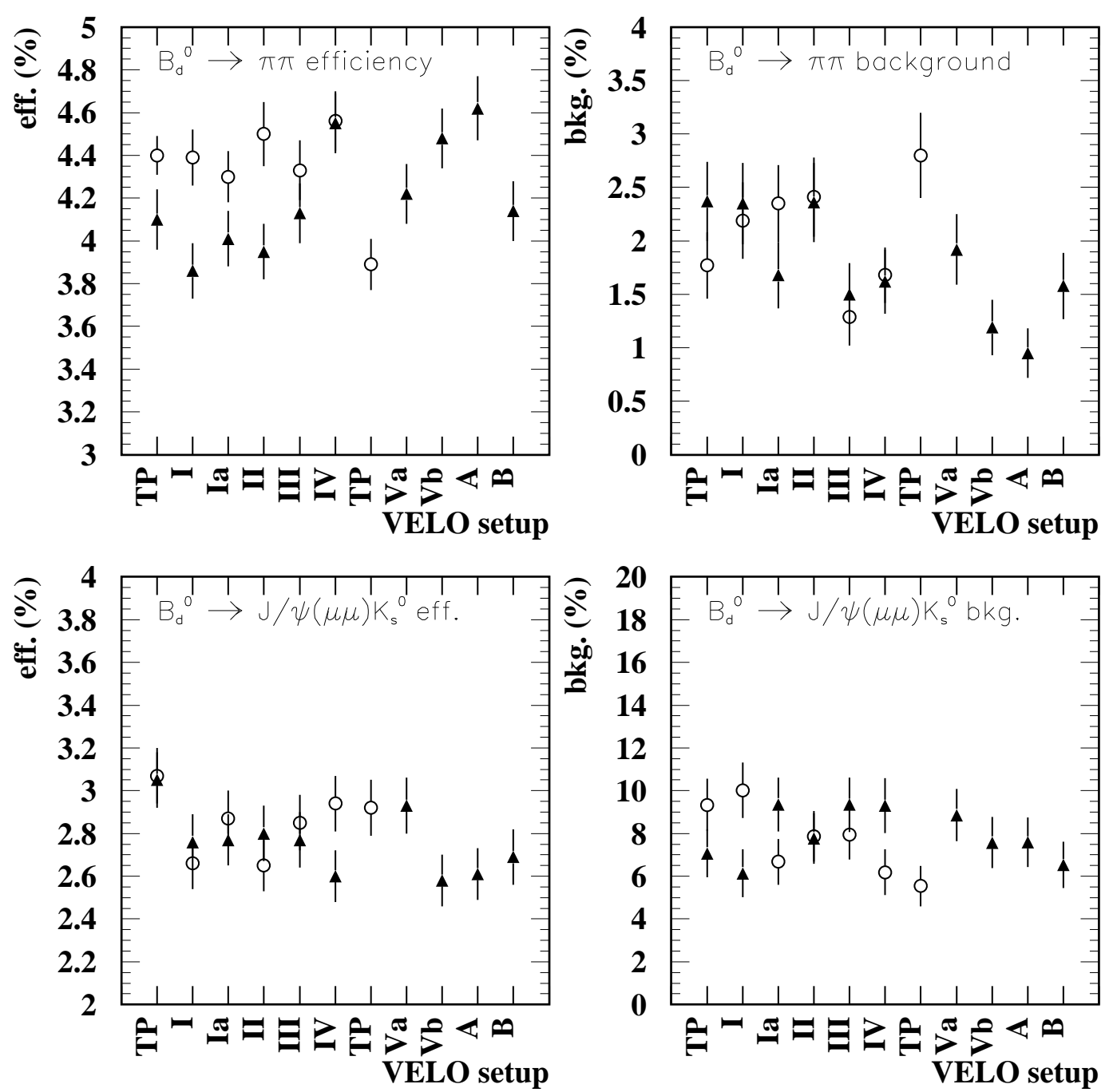

No large systematic effects, cuts not tuned for each design 


\section{Simulation of new VELO designs (cont'd)}

TP and baseline (backup) comparisons:

a) resolutions:

$\mathrm{PV}$ resolution $-5 \%$ worse

SV resolution $-15 \%$ better (no change)

decay length resolution -

$20 \%$ better (no change)

- strip pitch, Si inner radius

+ Si thickness, RF thickness

b) multiplicities:

charged multiplicity up $7 \%$

neutral multiplicity up $0.4 \%$

number of hits in other dets. - up 5\%

+ Si thickness, RF thickness 


\section{Simulation of new VELO designs (cont'd)}

c) number of high IP tracks (L1):

IP $>50,100 \mu \mathrm{m}$ up $10(20) \%(B \rightarrow \pi \pi)$

IP $>50,100 \mu \mathrm{m}$ up $20(40) \%$ (min. bias)

+ Si thickness, RF thickness and design

- strip pitch, Si inner radius

d) number of VELO hits per track - up 50\%

number of stations

e) LO efficiency - down 7\%

RF design

f) particle densities at min. radius - up 50\%

Si inner radius 


\section{Outlook}

Chosen new design is "baseline", with a conservative "backup" design (thicker Si, larger strip-pitch)

Main points:

a) need for increased RF shielding met

b) number of stations increased to $25-$ average number of VELO hits per track $50 \%$ higher $\rightarrow$ greater standalone tracking capability for the VELO

c) $B$ decay length resolution improved

d) slight increase in material budget

e) $B$ decay selection performance maintained

f) number of high IP tracks (for the L1 trigger) close to the TP values 